\title{
PSO based TCSC Placement for Security Enhancement
}

\author{
K. Kavitha \\ Assitant professor \\ Dept. of Electrical Engg. \\ Annamalai University
}

\author{
R.Neela, PhD. \\ Professor \\ Dept. of Electrical Engg. \\ Annamalai University
}

\begin{abstract}
Flexible AC Transmission System (FACTS) devices play a vital role in improving the static as well as dynamic performance of the power system. The location and size of these devices play a major role in deciding the extent to which the goal of improving the system performance is achieved in a cost effective manner. In this work, an objective function comprising the cost, line loadings and load voltage deviations is proposed, weights assigned to them decide their relative importance. PSO technique is applied for solving the problem and the effectiveness of the proposed method is tested on IEEE 14, 30 and 57 bus systems.
\end{abstract}

\section{Keywords}

FACTS devices, Thyristor Controlled Series Capacitor (TCSC), Particle Swarm Optimization (PSO), Security enhancement.

\section{INTRODUCTION}

Modern power systems are highly complex interconnected systems, so it is essential to improve the electric power utilization while maintaining the reliability and security. Due to increase in load demand, the magnitude of the power flows in some of the transmission lines are well above their normal limits and in some other lines, it is below their normal. Its overall effect will detoriote the voltage profiles and decrease the security of the power system. To meet the increasing load demand and satisfy the stability and reliability criteria, either existing transmission and generation facilities must be utilized more efficiently or new facilities should be added to the power system. Due to the constraints such as lack of investment and difficulties in getting new transmission line right -of-ways, the later is often difficult but the former can be achieved by using FACTS controllers[1].

FACTS (Flexible Alternating Current Transmission System) is a concept introduced by N.G.Hingorani[2]. FACTS technology opened up new opportunities for controlling power flow and enhancing the usable capacity of present, as well as new and upgraded lines. FACTS devices can effectively control the load flow distribution, improve the usage of existing system by increasing transmission capability, compensate for reactive power and improve stabilities of the power network. The possibility of controlling power flow in an electric power system without generation rescheduling or topological changes can improve the performance considerably [3]. However to achieve such benefits it is highly important to determine the suitable location and capacity of FACTS devices in the power system [4].

TCSC (Thyristor Controlled Series Capacitor) is a type of series compensator, that can provide many benefits for a power system including control of power flow in the line, damping power oscillations and mitigating sub synchronous resonance [5].TCSC is a variable impedance type series compensator. It consists of a series compensating capacitor shunted by a thyristor controlled reactor. By controlling the firing angle of thyristor, TCSC can change the line reactance smoothly and rapidly. TCSC has one of the two possible characteristics either capacitive or inductive by increasing or decreasing the reactance of the line $X_{l}[6]$. Moreover to avoid the over compensation of the line, the maximum values of capacitance and inductance are fixed at $-0.8 X_{l}$ and

$0.2 X_{l}$ [7]. World's first 3 phase, $2 * 165$ MVAR, TCSC was installed in 1992 in Kayenta substation, Arizona. It raised the transmission capacity of transmission line by $30 \%$ and effectively damped electromechanical power oscillations [8]. Optimal placement of TCSC is essential to tap the maximum benefits in terms of system performance and cost effectiveness.

A loss sensitivity index with respect to the control parameters of FACTS devices has been suggested and with the computed loss sensitivity index, the FACTS devices are placed on the most sensitive bus or line [9]. Fuzzy based approach for the optimal placement of FACTS device for enhancing the system security under normal and network contingencies has been discussed in [10]. The optimal location of a given number of FACTS devices is a problem of combinatorial analysis. To solve such kind of problems, heuristic methods can be used [11]. They permit to obtain acceptable solutions within a limited computation time. The application of Genetic Algorithm for the optimal location of multi type FACTS devices in order to maximize the system loadability is analysed in [12]. A Differential Evolution based algorithm to decide the optimal location and device rating has been suggested in [13] with an objective of enhancing the system security under single line contingencies. The Particle Swarm Optimization (PSO) is applied for the optimal location of FACTS devices to achieve minimum cost of installation and to improve system loadability, by considering thermal limit for the lines and bus voltage limit for the load buses as constraints [14]. Sensitivity analysis approach for finding the optimal location and PSO for the optimal parameter setting of TCSC has been suggested in [15] so as to maximize the loadability.

As bus voltage deviations on the load buses are also considered as appropriate indices that reflect the system security, these voltage deviations along with the cost of installation of the devices and the line loading are used to formulate the objective function that is to be minimized and the PSO technique is used to solve the problem in this work. 


\section{PROBLEM FORMULATION}

\subsection{Objective of the optimization}

As the cost of the FACTS devices, especially TCSC is high, in order to achieve the maximum benefit, the devices are to be installed at the optimal locations. Minimizing the cost of installation of the TCSC is chosen as the primary objective and the objective function is augumented with two indices, one for the load voltage deviation and the other for line loading thereby making it a comprehensive one, whose minimization leads to a cost effective, security oriented solution.

The objective function is formulated as

$\operatorname{Min} F=W_{1}\left[C_{T C S C} * S\right]+W_{2}[L V D]+W_{3}[L L]$

$F$ is the objective function;

$C_{T C S C}$ is the cost of TCSC device in US $\$ / \mathrm{KVar}$;

$S$ is the operating range of TCSC;

$L V D$ is the Load voltage deviation;

$L L$ is the Line loading;

$W_{1}, W_{2} \& W_{3}$ are the weight factors.

(i) $\operatorname{Cost}\left(C_{T C S C}\right)$

The first term of the objective function $C_{T C S C}$ presents the installation cost of TCSC device in the network, which is given by the following equation.

$$
C_{T C S C}=0.0015 s^{2}-0.7130 s+153.75
$$

(ii) Load voltage deviation $(L V D)$

Excessive high or low voltages can lead to an unacceptable service quality and can create voltage instability problems. FACTS devices connected at appropriate locations play a leading role in improving voltage profile thereby avoiding voltage collapse in the power system. The second term considered represents the load voltage deviations in order to prevent the under or over voltages at network buses.

$L V D=\sum_{m=1}^{n b}\left(\frac{V_{\text {mref }}-V_{m}}{V_{\text {mref }}}\right)^{n}$

$V_{m}$ is the voltage magnitude at bus $\mathrm{m}$

$V_{m r e f}$ is the nominal voltage at bus $\mathrm{m} \&$ is considered as 1.0 pu.

$m$ refers to the load buses, where $V_{m}$ is less than $V_{m r e f}$.

(iii) Line loading $(L L)$

TCSC is located in order to remove the overloads and to distribute the load flows uniformly. To achieve this, line loading is considered as the third term in the objective function.
$L L=\sum_{l=1}^{n l}\left(\frac{S_{l}}{S_{l \max }}\right)^{n}$

$S_{l}$ is the apparent power in the line $l$.

$S_{l \max }$ is the apparent power rating of line $l$.

\subsection{The optimization variables}

are

The optimization variables considered in this work

(a) The number of TCSC devices to be installed is taken as the first variable.

(b) TCSC location is considered as the second variable to be optimized. TCSC's are not installed in the lines where the transformers exist.

(c) The reactance of the TCSC is considered as the third variable. The working range of TCSC is considered as follows.

$-0.8 X_{l} \leq X_{T C S C} \leq 0.2 X_{l}$

$X_{\text {TCSC }}$ is the reactance added to the line by placing TCSC.

$X_{l}$ is the reactance of the line where TCSC is located.

\section{OVERVIEW OF PSO TECHNIQUE}

Particle swarm optimization is a heuristic search technique developed by Eberhart and Kennedy[16] based on the concept of swarm intelligence exhibited by the flock of birds, school of fish etc in which each member of the group adjusts its behavior based upon its own experience and the experience of the swarm. .This sort of social behavior is used to simulate the problem solving environment in which a swarm is randomly generated in terms of solution variables of the problem. The individuals in a swarm are called particles. After generating the swarm, the fitness values of the particles $P_{\text {best }}$ are evaluated and compared against the values obtained from the previous iteration. The particles with the best values of fitness function in the next generation $P_{b e s t}$ are retained. $G_{\text {best }}$ is the best value attained so far by the swarm of particles. In each iteration, $G_{b e s t}$ of the current swam is compared with the $G_{b e s t}$ of the previous iteration and whichever is lower is retained along with the corresponding particle.

The position update of particles is carried out through the expression (6) in which the velocity is calculated using (7)

$$
\begin{aligned}
& X_{i d}^{k+1}=X_{i d}^{k}+V_{i d}^{k+1} \\
& V_{i d}^{K+1}=W V_{i d}^{K}+c_{1} r_{1}\left(P_{b e s t i d}^{k}-X_{i d}^{k}\right)+c_{2} r_{2}\left(G_{b e s t i d}^{k}-X_{i d}^{k}\right)
\end{aligned}
$$

The inertia weight in (7) is calculated using the following expression. 
$W=W_{\text {max }}-\frac{W_{\text {max }}-W_{\text {min }}}{\text { iter }_{\text {max }}} *$ iter

This iterative procedure is repeated till a specified number of swarms is reached or until a predefined amount of time has elapsed or until there is no considerable difference between the outcomes of any two subsequent iterations.

$V_{i d}^{k+1}$-Velocity of the $i^{t h}$ individual at $(k+1)^{t h}$ iteration.

$V_{i d}^{k}$-Velocity of the $i^{t h}$ individual at $k^{\text {th }}$ iteration

$X_{i d}^{k}$ - Position of the $i^{t h}$ individual at $k^{\text {th }}$ iteration.

$X_{i d}^{k+1}$ - Position of the $i^{t h}$ individual at $(k+1)^{t h}$ iteration.

$P_{\text {bestid }}$ - Best position of the $i^{\text {th }}$ individual.

$G_{\text {bestid }}$ - Best position among the individuals.

$r_{1}, r_{2}$ - Random numbers distributed within the interval $[0,1]$

$c_{1}, c_{2}$ - Positive constants called acceleration constants.

$W$ - Inertia weight.

$W_{\max }$ - Initial value of inertia weight.

$W_{\min }$ - Final value of inertia weight.

iter $_{\text {max }}$ - Maximum number of iterations.

iter - Current iteration number.

$d=1,2, \ldots . . D, D$ is the number of members in a particle.

$i=1,2, \ldots \ldots . m, m$ is the size of the swarm.

\section{ALGORITHM}

The algorithm of the proposed work is explained below.

Step1: The system data and the load factor are initialized.

Step2: PSO parameters such as the size of swarm $\mathrm{m}$, maximum number of iterations, the number of variables to be optimized, limits of each variables in the particle, $c_{1} \& c_{2}$ values, $W_{\min } \& W_{\max }, \mathrm{D}$, velocity limits, $P_{\text {best }}$ and $G_{\text {best }}$ are initialized.

Step3: An initial population is randomly generated considering the variables to be optimized. [the number of TCSCs, location of TCSC, parameter setting of TCSC]

Step4: For each particle i $[i=1,2, \ldots . . \mathrm{m}]$ in the population, the objective function is evaluated.

Stpe5: The calculated value of each particle is compared with its $P_{b e s t}$ and $P_{b e s t}$ of each particle is updated.

Step6: $G_{b e s t}$ is calculated, then compared with the $G_{b e s t}$ in the previous iteration and it is updated.
Step7: A new population is created by changing the velocity and position of the particle.

Step8: If stopping criterion is satisfied, the best individual is printed, else repeated from step 4.

Step9: The same procedure is repeated for different load factors.

\section{SIMULATED RESULTS \\ 5.1. Case (1) 14 bus system}

Table1: Line loading for different load factors

\begin{tabular}{|l|l|l|l|l|l|l|}
\hline Load factor & 1 & 1.1 & 1.2 & 1.3 & 1.4 & 1.5 \\
\hline Without TCSC & 17.5892 & 19.2093 & 20.9319 & 22.4464 & 24.0519 & 25.7766 \\
\hline With TCSC & 17.3907 & 19.0305 & 20.6862 & 22.3651 & 23.3228 & 25.0535 \\
\hline
\end{tabular}

\begin{tabular}{|l|l|l|l|l|l|l|}
\hline With TCSC & 17.3907 & 19.0305 & 20.6862 & 22.3651 & 23.3228 & 25.0535 \\
\hline
\end{tabular}

Table2: Line voltage deviation for different load factors

\begin{tabular}{|l|l|l|l|l|l|l|}
\hline Load factor & 1 & 1.1 & 1.2 & 1.3 & 1.4 & 1.5 \\
\hline Without TCSC & 0.3509 & 0.3696 & 0.4075 & 0.4875 & 0.5689 & 0.5922 \\
\hline With TCSC & 0.2884 & 0.3079 & 0.3275 & 0.3479 & 0.3801 & 0.4024 \\
\hline
\end{tabular}

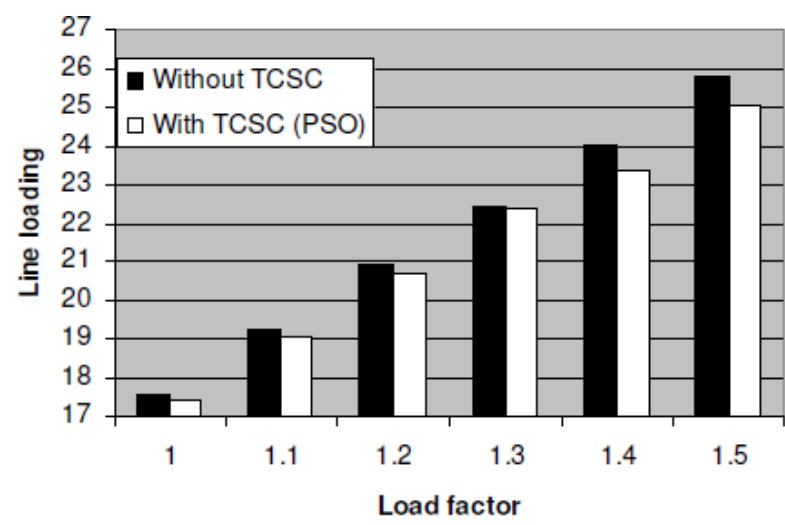

Fig. 1: Line loading VS percentage of load

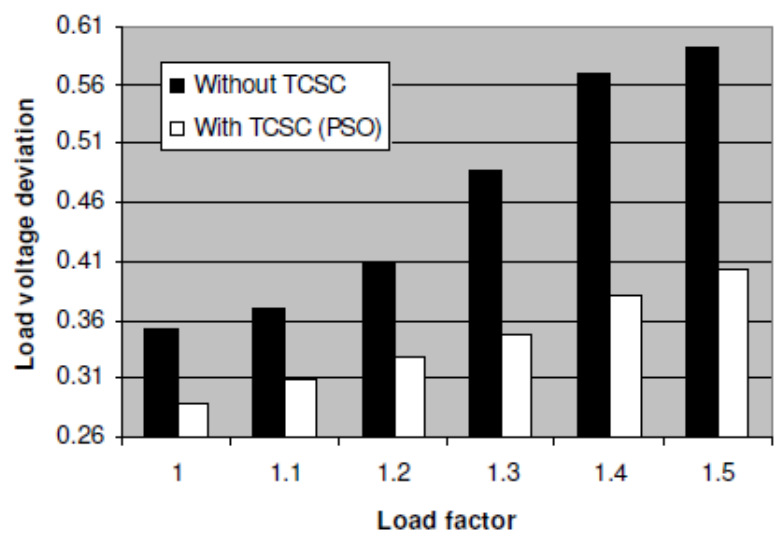

Fig. 2: Load voltage deviation VS percentage of load

\subsection{Case (2) 30 bus system}

Table3: Line loading for different load factors

\begin{tabular}{|c|c|c|c|c|c|c|}
\hline Load factor & 1 & 1.1 & 1.2 & 1.3 & 1.4 & 1.5 \\
\hline Without TCSC & 14.5592 & 16.2116 & 17.9504 & 19.7258 & 21.5352 & 23.1761 \\
\hline With TCSC & 14.5522 & 16.1973 & 17.9397 & 19.7155 & 21.5319 & 23.1159 \\
\hline
\end{tabular}


Table4: Line voltage deviation for different load factors

\begin{tabular}{|c|c|c|c|c|c|c|}
\hline Load factor & 1 & 1.1 & 1.2 & 1.3 & 1.4 & 1.5 \\
\hline Without TCSC & 0.6967 & 0.6974 & 0.7145 & 0.7342 & 0.7548 & 0.7834 \\
\hline With TCSC & 0.6965 & 0.6973 & 0.7128 & 0.7335 & 0.7529 & 0.7578 \\
\hline
\end{tabular}

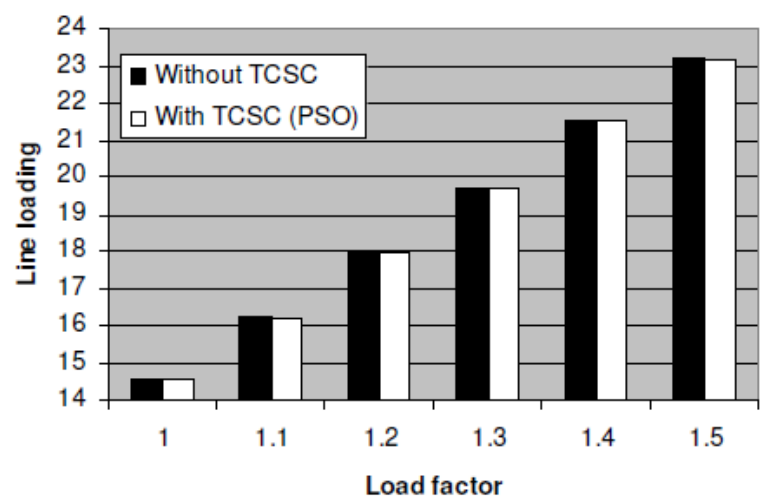

Fig. 3: Line loading VS percentage of load

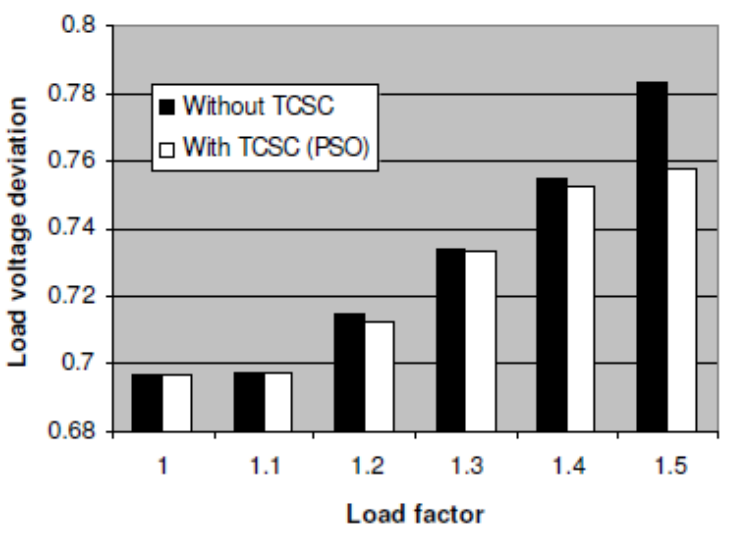

Fig. 4: Load voltage deviation VS percentage of load

\subsection{Case (3) 57 bus system}

Table5: Line loading for different load factors

\begin{tabular}{|l|c|c|c|c|c|c|}
\hline Load factor & 1 & 1.1 & 1.2 & 1.3 & 1.4 & 1.5 \\
\hline Without TCSC & 53.33 & 61.29 & 70.01 & 79.86 & 92.01 & 105.16 \\
\hline With TCSC & 53.26 & 61.23 & 69.92 & 79.66 & 91.59 & 104.23 \\
\hline
\end{tabular}

Table6: Line voltage deviation for different load factors

\begin{tabular}{|l|c|c|c|c|c|c|}
\hline Load factor & 1 & 1.1 & 1.2 & 1.3 & 1.4 & 1.5 \\
\hline Without TCSC & 3.88 & 4.16 & 4.46 & 4.46 & 5.97 & 6.88 \\
\hline With TCSC & 3.86 & 4.16 & 4.45 & 4.46 & 5.65 & 6.78 \\
\hline
\end{tabular}

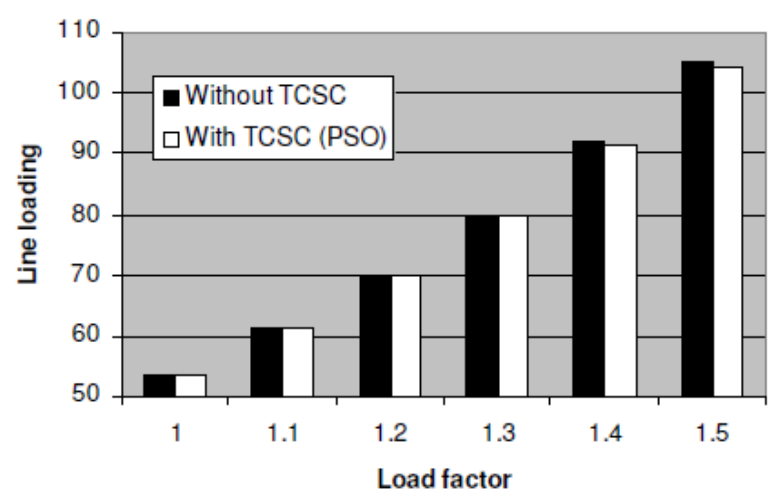

Fig.5: Line loading VS percentage of load

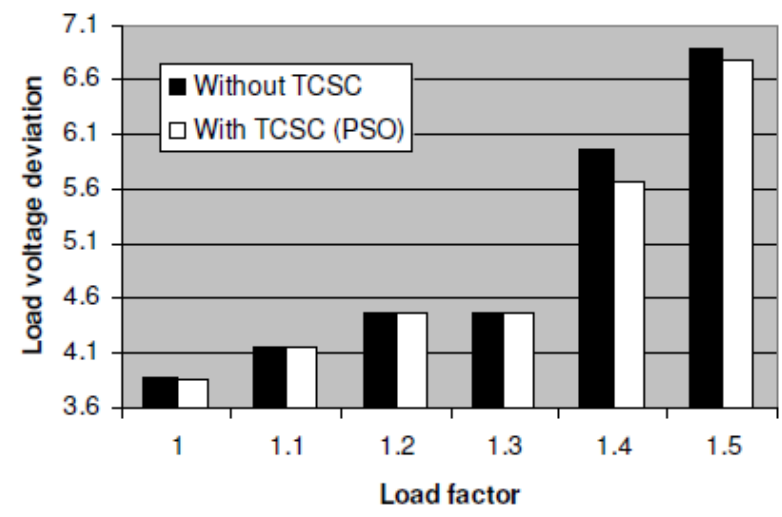

Fig. 6: Load voltage deviation VS percentage of load

The effectiveness of the proposed algorithm has been tested on IEEE 14, 30 and 57 bus systems. As line loadings form a part of the objective function, overload conditions are simulated by simultaneously varying the real and reactive powers on the load buses keeping the load power factor constant. Minimizing the installation cost of the devices forms the other objective and this is achieved by optimally sizing and placing the devices. Moreover the maximum number of devices is limited to 2 on a 14 bus system, 3 on a 30 bus system and 5 on a 57 bus system.

Under over loaded conditions, the increased voltage drops on transmission lines result in unsatisfactory voltage profile. So, reduction in the deviation of load voltages is made as a third objective.

Figures 1 and 2 show the variation of line loadings and load voltage deviations for 14 bus system with and without TCSC in the form of double bar charts. It has been observed that line loadings have reduced considerably under overloaded conditions and the load voltage deviations are much lesser when the system is operated with TCSCs at optimum locations.

In the 30 bus system, though load voltage deviations vary only marginally after the installation of TCSC, there is a considerable reduction in line loadings as illustrated in Figures 3 and 4

In 57 bus system, both parameters show improvement in the positive direction similar to that of 14 and 30 bus systems, strongly reinforcing the FACT that TCSC placement improves the load flows and enhances the voltage distributions under normal and overloaded conditions. 


\section{CONCLUSION}

PSO technique has been applied for solving the problem of security enhancement of power systems. Though TCSCs can be placed at any feasible location in the power system, their locations and ratings are to be fixed optimally as they turn out to be costlier than conventional compensating devices. Here the problem of device placement is guided through the PSO algorithm which gives the solution for the comprehensive objective function consisting of cost of the device, load voltage deviations and line loadings. The proposed method yields an efficient solution which considerably reduces load voltage deviations and realize the lines of their over loads under various load conditions.

\section{REFERENCES}

[1] Surekha Manoj, Dr. Puttaswamy P.S., "Importance of FACTS Controllers in power systems", International Journal of Advanced Engineering Technology, vol.II, Issue III, july-sep, 2011, pages 207-212.

[2] N.G.Hingorani and L.Gyugyi, "Understanding FACTS", IEEE Press, 1999.

[3] S.N.Singh, A.K.David, "Optimal location of FACTS devices for congestion management", Electric Power Systems Research vol 58, 2001, pages 71-79.

[4] R.Benabid, M.Boudour, M.A. Abido, "Optimal location and setting of SVC and TCSC devices using nondominated sorting Particle Swarm Optimization", Electric Power Systems Research vol 79, 2009, pages 1668-1677.

[5] Hugo Ambriz-Perez, Enrique Acha, Claudio R. FuerteEsquivel, "TCSC-firing angle model for optimal power flow solutions using Newton's method", Electric Power Systems Research vol 28, 2006, pages 77-85.

[6] Naresh Acharya, N.Mithulanathan, "locating series FACTS devices for congestion management in deregulated electricity markets", Electric Power Systems Research vol 77, 2007, pages 352-360.

[7] Ghamgeen I.Rashed, Yuanzhang Sun, Kai-Pei Liu, "Optimal placement of Thyristor Controlled Series Compensation in Power System based on Differential Evolution Algorithm" IEEE, seventh International Conference on Natural Computation, 2011.
[8] S.Meikandasivam, Rajesh Kumar Nema, Shailendra Kumar Jain, " Behavioral Study of TCSC device- A matlab / simulink Implementation " World Academy of Science, Engineering and Technology, 2008

[9] Preecha Preedavichit, S.C. Srivastava,"Optimal reactive power dispatch considering FACTS devices" Electric Power Systems Research vol 46, 1998, pages 251-257.

[10] K.Visaka, D.Thukaram, Lawrence Jenkins, "Application of UPFC for system security improvement under norma and network contingencies" electric power systems research-2003.

[11] Sung-Hwan Song, Jung-Uk Lim, Seung-II Moon, "Installation and operation of FACTS devices for enhancing steady -state security" Electric Power Systems Research vol 70, 2004, pages 7-15.

[12] Stephane Gerbex, Rachid Cherkaoui, Alain J. Germond, "Optimal location of multi-type FACTS devices in a power system by means of genetic algorithms" IEEE Transactions on power systems, vol. 16. Aug. 2001.

[13] Husam I.Shaheen, Ghamgeen I.Rashed, S.J. Cheng, "Optimal location and parameter setting of UPFC for enhancing power system security based on differential evolution algorithm", Electric Power Systems Research vol 33, 2010, pages 94-105.

[14] M.Saravanan, S.Mary Raja Slochanal, P.Venkatesh, J.Prince Stephen Abraham, "Application of particle swarm optimization technique for optimal location of FACTS devices considering cost of installation and system loadability", Electric Power Systems Research vol 77, 2007, pages 276-283.

[15] K.satyanarayana, B.K.V.Prasad, G.Devanand, N.Siva Prasad, "optimal location of TCSC with minimum installation cost using PSO", International Journal of computer science and technology, vol 2, dec 2011.

[16] James Kennedy, Russell Eberhart "Particle Swarm Optimization", in Proceedings of IEEE International Conference on Neural Networks, vol14, pp19421948,1995 